\title{
La construction allégorique de Caresme et la représentation de la faim dans les débats de Caresme et Charnage (XIII ${ }^{\mathrm{e}}-\mathrm{XV}^{\mathrm{e}}$ siècles)
}

\section{Laëtitia Tabard}

\section{(2) OpenEdition}

\section{Journals}

Édition électronique

URL : http://journals.openedition.org/questes/2729

DOI : 10.4000/questes. 2729

ISSN : 2109-9472

\section{Éditeur}

Les Amis de Questes

\section{Édition imprimée}

Date de publication : 15 juin 2007

Pagination : 65-76

ISSN : 2102-7188

\section{Référence électronique}

Laëtitia Tabard, «La construction allégorique de Caresme et la représentation de la faim dans les débats de Caresme et Charnage (XIIIe-XVe siècles) », Questes [En ligne], 12 | 2007, mis en ligne le 15 janvier 2014, consulté le 19 avril 2019. URL : http://journals.openedition.org/questes/2729; DOI : 10.4000/questes.2729 


\title{
La construction allégorique de Caresme et la représentation de la faim dans les débats de Caresme et Charnage (XIII ${ }^{\mathrm{e}}-\mathrm{XV}^{\mathrm{e}}$ siècles)
}

\author{
Laetitia TABARD
}

Le conflit entre Caresme et Charnage apparait dans la littérature au XIII ${ }^{\mathrm{e}}$ siècle et inspire pièces et poèmes jusqu'au $\mathrm{XVIII}^{\mathrm{e}}$ siècle ${ }^{1}$. Pour la période médiévale, on trouve trois textes français, un anonyme daté de la seconde moitié $\mathrm{du} \mathrm{XIII}^{\mathrm{e}}$ siècle qui s'intitule La Bataille de Caresme et de Charnage ${ }^{2}$, une ballade d'Eustache Deschamps dont le refrain est « Maudis soit-il, et benoit soit Charnaige $»^{3}$, et datée approximativement de 1380, et une pièce en prose de 1490, imprimée sous le titre La Dure Et Cruelle Bataille et paix du glorieux sainct Pensart a l'encontre de Caresme ${ }^{4}$. Cet ensemble relativement restreint de textes a exercé une influence certaine sur d'autres débats, en contribuant à dessiner une division symbolique du monde 5 .

\footnotetext{
${ }^{1}$ Pour un inventaire des œuvres, voir Martine GrinBerg, et Sam Kinser, « Les Combats de Carnaval et de Carême. Trajets d'une métaphore. ", Annales Economies, Sociétés, Civilisations, Paris, Armand Colin, 38:1 (1983), p. 65-98. Le thème s'épanouit au XVI siècle, et l'on relève au début du siècle un poème narratif, Le merveilleux Conflit entre Caresme et Charnage, et une pièce versifiée attribuée à Jehan D'Abondance, Le Testament de Carmentrant.

${ }^{2}$ La Bataille de Caresme et de Charnage, éd. Grégoire LozInSKI, Paris, Honoré Champion, 1933.

${ }^{3}$ Euvres complètes de Eustache Deschamps, éd. Marquis de QueuX DE SAINT-HILAIRE, Paris, Firmin-Didot, 1878-1903, tome III, Ballade 350, p. 75-77.

${ }^{4}$ La Dure Et Cruelle Bataille et paix du glorieux sainct Pensart a l'encontre de Caresme, dans Jean-Claude Aubailly, Deux Jeux de carnaval de la fin du Moyen Âge, Genève, Droz, 1978, p. $1-71$.

${ }^{5}$ On trouve des échos de cette tradition par exemple dans deux débats de Jean Molinet, Le Débat d'Avril et de May, et Le Débat de la Chair et du Poisson, ainsi que dans les débats qui évoquent la nourriture ou bien les divisions du temps comme ceux de l'eau et du vin, ou de l'hiver et de l'été.
} 
Ces œuvres construisent une représentation de la faim en l'opposant fondamentalement à l'appétit. Leur propos est en effet intimement lié aux rites alimentaires du carême et de son envers, le Mardi gras; entre la période où il est autorisé de manger de la viande et d'avoir des relations sexuelles, et qui s'achève sur une fête qui célèbre la bonne chère, et un régime alimentaire dit « maigre » où l'on se nourrit une seule fois par jour, essentiellement de poisson et de légumes, selon les prescriptions religieuses, l'opposition est tranchée. Elle est figurée littérairement par le débat et la bataille entre Caresme et Charnage, s'achevant sur la victoire de la seconde entité. La faim y est opposée à l'appétit du bon vivant qui mange gras, et on tend à la distinguer du désir de se nourrir, son symptôme le plus reconnaissable, pour n'en retenir que l'idée d'un manque.

La représentation d'une idée aussi singulièrement négative n'est pas sans difficultés. La figuration du jeûne passe par la forme allégorique, donc par un personnage, allégorie paradoxale qui est censée incarner du négatif, et donner une chair à l'idée du manque. Cette représentation, de plus, ne va pas de soi dans la tradition de la Psychomachia dont relève le conflit entre Caresme et Charnage. Le combat entre les vices et les vertus oppose la gloutonnerie, le péché de gourmandise, et la vertu de Tempérance et de «Souffisance ». Dans cette perspective morale, la faim vécue comme une souffrance physique n'a guère sa place. Comment dès lors parler de la faim, et construire une allégorie à partir de l'idée du manque, sans références culturelles stables?

\section{La parodie de l'épopée et la faim comme mauvais régime}

Charnage et Caresme sont modelés à partir du motif épique des deux seigneurs en guerre. Le premier personnage relève de l'image traditionnelle du prince libéral, qui apporte l'abondance et suscite la reconnaissance. Toujours représenté entouré de bons amis, et accueilli avec joie par ses gens, béni par Mardi-Gras chez Deschamps, Charnage fait l'objet d'éloges appuyés dans les 
salutations comiques auxquelles se livrent ses partisans dans le jeu de Carnaval : son messager le nomme « Charnau notre vray nourrisson /Et emplisseur de nos entrailles », et son ambassadeur, maître Accipé : «Charnau grant ami de Nature /Vray nourrisson de ses boyaulx. $\rangle^{6}$ Ainsi l'appétit est figuré par la joie manifestée à la vue d'un véritable ami. L'insistance sur le boyau plein rappelle que seul Charnage rassasie ses fidèles, contrairement à son adversaire.

La Bataille de Caresme et de Charnage fait également de Caresme un puissant seigneur féodal, mais dans une opposition négative avec Charnage. D’emblée, Caresme est présenté comme un affameur :

Li autres qui maintient la guerre

Contre Charnage le baron, A non Quaresme le felon, Qui moult est fel et anïeus :

Ce sevent bien li fameilleus Qui ont esté en son païs.

Précisément, Caresme est ici un traître, ce qui se comprend par opposition au « vray nourrisson » qu'est Charnage : il dispense une nourriture trompeuse, qui ne satisfait nullement aux besoins de ses sujets. Les effets de Caresme se conçoivent dans cette différence entre son apparence agréable et la réalité du régime auquel il soumet son peuple :

De povres gens est moult haïs :

Moult le heent la gent menue !

Les riches hommes biau salue

Et honneure et fet bele chiere

Et la povre gent met arriere. ${ }^{8}$

La faim est figurée ici par le mauvais traitement infligé par le seigneur, vécu comme une brimade injuste, et peut-être faut-il aussi y voir un signe de l'effet très différent des privations selon les classes sociales.

${ }^{6}$ La Dure Et Cruelle Bataille..., éd. cit., v. 177-178, p. 13, et v. 982, p. 60.

${ }^{7}$ La Bataille de Caresme et de Charnage, éd. cit., v. 32-37, p. 2-3.

${ }^{8}$ Ibid., v. 38-42, p. $2-3$. 
On retrouve cette nuance dans la ballade d'Eustache Deschamps, qui n'oppose plus pauvres et riches, mais distingue trois degrés dans la privation selon les trois ordres de la société ; Caresme prend aux pauvres, qui n'ont déjà pas assez et qui de surcroît travaillent :

Caresme met les povres gens au bas, Jeuner les fait et estre mal servis, Et les contraint par griefs labours de bras (...). ${ }^{9}$

La faim ressemble ici à la corvée imposée par le maître. En revanche, Carême ne fait qu'appauvrir le régime des religieux :

Toudis leur fait june et afflictions, Et a pluseurs tenir povre mesnaige, $(\ldots) .{ }^{10}$

Enfin, il se contente de vider ceux qui étaient bien nourris :

Aux bien peüz fait avoir ventres plas Il vuide ceuls que j'avoie remplis. ${ }^{11}$

Le conflit entre le bon et le mauvais prince (le «tirant $»^{12}$ selon le mot d'Eustache Deschamps) place Caresme du côté de l'avarice, en opposition avec la libéralité de Charnage, et l'intègre donc au système des vertus et des vices. Mais l'analogie qui permet de figurer la différence entre les deux régimes alimentaires confère une portée politique au discours sur la faim : le mauvais régime est le mauvais gouvernement du seigneur qui maltraite ses sujets.

La critique politique se réduit à quelques allusions, et ce n'est pas l'essentiel dans ces textes où l'effet comique prime manifestement, mais elle permet de donner sens à la victoire de Charnage sur son adversaire. La Bataille de Caresme et de Charnage raconte en effet, en parodiant le modèle épique, la victoire sur la faim par la défaite de Caresme, mais il faut se garder de

${ }_{9}^{9}$ Euvres complètes de Eustache Deschamps, éd. cit., Ballade 350, v. 11-13.

${ }^{10}$ Ibid., v. 27-28.

${ }^{11}$ Ibid., v. 31-32.

${ }^{12}$ Ibid., v.19. 
l'interpréter de manière univoque comme une revanche des appétits et de la licence: il s'agit, dans la métaphore politique comme dans l'arrière-plan alimentaire, de restaurer un ordre du monde. La dispute commence sur une question de préséance; les Français semblent préférer le poisson, et Charnage s'estime lésé. Ce renversement contre-nature impose une remise en ordre symbolique. C'est l'objet du récit dans son ensemble que de construire la figure négative de Caresme, en départageant Caresme et Charnage, et en attribuant aux deux seigneurs un territoire et un temps définis. À l'issue du combat, Charnage, aidé de Noël, est vainqueur et s'approprie les poissons : Caresme est réduit à néant et Charnage s'érige en incarnation de la gourmandise. La Bataille de Caresme et de Charnage fonctionne ici comme une épopée fondatrice, qui façonne la figure du jeûne en lui enlevant ses attributs, composant une sorte d'allégorie par le vide, figurant la faim.

\section{La parodie de l'allégorie et la symbolique des aliments}

La Bataille de Caresme et de Charnage relève également de la parodie de la bataille allégorique, telle qu'on la trouve dans La Psychomachia de Prudence, mais la bataille des vices et des vertus se transforme de manière comique en bataille de viandes et de poissons, personnifiés en combattants.

Caresme domine une grande partie de la terre, et règne sur la mer et les rivières, c'est-à-dire sur l'espace où l'on trouve la nourriture autorisée pendant la période de jeûne; poissons et légumes constituent donc son armée. On peut cependant s'étonner que, dans La Bataille de Caresme et de Charnage, Caresme soit représenté par d'appétissants plats :

La menuise est el premier front ;

Les anguilles au brouet sont

Après a bataille rengie ;

Harens fres a la blanche aillie

Viennent aprés, et li mulet, 
Hadoc et merlenc et rouget

Et tant de ces autres poissons

Viennent poingnant a esperons $(\ldots) .{ }^{13}$

L'énumération de victuailles parodie l'énumération des troupes et l'effet d'amplification qui caractérisent la chanson de geste, et donne la sensation de l'abondance. La représentation des troupes de Caresme se révèle donc curieusement alléchante, et produit l'inverse de l'effet attendu.

L'opposition des deux armées se creuse lors de la préparation au combat où chaque camp se pare de ses attributs symboliques. On trouve alors une opposition plus nette entre le gras et le maigre, notamment si l'on compare les descriptions des armures des deux seigneurs. Pour Caresme :

Son hauberc fu d'un fres saumon,

De lamproie son auqueton ;

Si vous di que ses espaulieres

Furent de granz plaïs raieres ;

De bon lus fu son elme fes;

Li cercle ne fu pas mauvés,

Qui estoient d'anguilles rosties.

Toutes ses armes a sesies.

Puis li a l'en çainte une espee,

D'une grant sole longue et lee.

Si vous di que ses esperon

Furent d'arestes de poisson.

Cheval li a l'en amené,

Un grant mulet bien enselé ; (...). ${ }^{14}$

L'image des poissons plats, longs et grands va s'opposer aux rondeurs et aux formes très diverses des éléments composant l'armement de Charnage, qui est décrit en regard du premier. L'opposition des deux régimes alimentaires passe alors par la distinction entre le plat et le rond, mais aussi entre le « fres » et le chaud, entre les aliments non préparés et ceux qui sont «ouvrés », cuisinés. Cependant Caresme ne se distingue pas de Charnage fondamentalement et la

${ }^{13}$ La Bataille de Caresme et de Charnage, éd. cit., v. 187-194, p. 8.

${ }^{14}$ Ibid., v. 281-295, p. 12-13. 
description, qui passe par les mêmes étapes, les rapproche. Certes, le plat du felon Caresme est trompeur, mais les deux régimes semblent également appétissants. L'exaltation de l'appétit et de la bonne chère contamine jusqu'à l'allégorie de la faim, qui donne effectivement l'eau à la bouche, le texte constituant une sorte d'entremet littéraire ${ }^{15}$, à moins que l'énumération de plats puisse s'interpréter précisément comme signe de la faim.

Les deux autres textes s'emploient davantage à creuser la différence entre les mets alléchants et la triste nourriture de Caresme. L'opposition n'est pas encore très nette à considérer les énumérations de mets que l'on trouve dans la ballade d'Eustache Deschamps à la fois pour Caresme et pour Charnage. Le poète évoque pour ce dernier « beufs, vaiches et moutons, / Veaulx et aigneaulx, connins, perdriz, chapons, / Cers et chevreaulx, pors, bieurre, œufs et frommaige, / Oes, malars, faisans, grues et paons "), et à la fin du poème «Porree au lart, pastez (...) / Connins, cabriz, oes, tartres et flaons ${ }^{16}$. Aucun ordre particulier ne semble prévaloir, mais la diversité des formes même évoque l'abondance et le spectaculaire ${ }^{17}$. Les plats de Caresme sont également énumérés, mais ils sont associés à leurs effets néfastes et à une présentation négligée : on «met devant », « en un tas $»^{18}$, des éléments simples. Les soupes et brouets servis à Caresme, dont le messager se nomme précisément «Humebrouet » dans le jeu de Carnaval, se présentent comme des plats liquides, épais, d'aspect indéfini et répugnant, que l'on « hume » d'un trait.

La gourmandise oriente au contraire vers le bon morceau, le « loppin », et même vers le petit morceau et la «bribe», comme l'indique le surnom de «bribeur» donné à Charnau. Les partisans du personnage font également

\footnotetext{
${ }^{15} \mathrm{Il}$ est possible que les textes soient directement liés à la pratique festive du banquet de fête : c'est l'hypothèse de Mikhaïl Bakhtine, qui considère que le débat est intimement lié au propos de table, et en constitue une mise en forme littéraire. Voir Mikhaïl BAKHTINE, L'œuvre de François Rabelais et la culture populaire au Moyen Âge et sous la Renaissance, Paris, Gallimard, 1990 (1 $1^{\text {ère }}$ éd. 1970), p.279.

${ }^{16}$ Euvres complètes de Eustache Deschamps, éd. cit., Ballade 350, v. 6-9 et v. 46-47.

${ }^{17}$ Le caractère spectaculaire peut expliquer la vogue de certains oiseaux comme le paon.

${ }^{18}$ Euvres complètes de Eustache Deschamps, éd. cit., Ballade 350, v. 16 et v. 23.
} 
allusion à plusieurs reprises à la gestuelle du gourmand, s'adonnant au péché de « lescherie », comme celle de se « lescher le doy /(...) De bons morceaulx », ou de se $«$ lescher les lippes $»^{19}$ : l'attitude du gourmand tient moins de la dévoration gloutonne que de l'acharnement minutieux à grignoter jusqu'aux plus petits morceaux. La prédilection pour les oiseaux, dans la ballade comme dans le jeu de Carnaval, révèle un goût particulier pour les aliments délicats et les morceaux fins, mais aussi pour une nourriture qui vaut comme symbole, se rattachant à l'élément aérien ${ }^{20}$; de même, sur un autre plan, le « connin », cité deux fois par Deschamps, est surtout le signe de la levée des interdits sexuels.

Les plats, qui ne sont plus personnifiés, semblent donc traversés par des lignes de rupture symboliques, opposant les préparations appétissantes et les aliments repoussants.

\section{La parodie de la moralité et la faim comme souffrance du corps}

Dans le jeu de Carnaval, les deux personnages eux-mêmes font l'objet d'une mise en scène plus précise, mais Caresme surtout évolue, en fonction de son adversaire, dont la représentation semble plus stable.

Les caractères plat, pointu, saillant, caractérisant les mets ou transposés métaphoriquement en armement, deviennent des attributs de Caresme. On trouve nettement l'association du personnage à la maigreur d'un corps décharné, et il a lui-même «eschine et mesgre doz » ${ }^{21}$. Ses partisans portent les noms évocateurs d'Humebrouet, Penceapoix, mais aussi de Vuydeboyau, Maisgredoz, Aliboron longue eschine.

Le prologue situe également les deux entités personnifiées dans un espace symbolique, qui parodie celui de la moralité : en haut se trouvent Bacchus et son

${ }^{19}$ La Dure Et Cruelle Bataille..., éd. cit., v. 496, p. 31, v. 205-207, p. 14-15, et v. 692, p. 42.

${ }^{20}$ Voir Liliane PlOUVIER, «L'Alimentation carnée au Haut Moyen Âge », Revue belge de philologie et d'histoire, 80:4 (2002), p. 1357-11369. L'auteur souligne ce lien entre hiérarchie des aliments et échelle des êtres (p. 1364).

${ }^{21}$ Ibid., v. 200, p. 14 
échansonnerie, et d'autre part la cuisine de Macquaire, sorte d'enfer. Quant aux protagonistes, ils sont décrits ainsi :

D'autre cousté, sur ce tonneau

Regardez moy la portraicture

Du grand bribeur notre Charnau

Qui porte une grasse ceinture.

Il mettra a desconfiture

Par Commentenon et par Chose

Caresme tout plain de froidure

Que voyez la qui se repose. ${ }^{22}$

Plus tard dans le texte, on mentionne que Caresme se trouve " en une chaise assis qui est garnie de merlus et harens soretz $\|^{23}$. C'est l'opposition symbolique entre boire et manger qui gouverne ici la distribution scénique, Caresme se trouvant du côté de la cuisine et de la table.

L'insuffisance des plats est signifiée de fait par la mauvaise qualité des aliments, et non par leur petite quantité. Dans la ballade d'Eustache Deschamps, « huile de chenevis », « Noix moysies, pommes et pain faitis », « Harens puanz, poissons de mer pourris $»^{24}$ se substituent à l'alimentation habituelle, et la puanteur devient le signe représentatif du plat de Caresme. Le jeu de Carnaval reprend comme un refrain ce motif: on se bat à coup de « raye toute puante " dans la bataille, et, une fois vaincu, Caresme fait apporter un plat de navets en signe de paix, ce qui constitue apparemment un jeu de mots, le navet étant réputé faire « vessir ».

Des plats, ce signe distinctif passe au personnage allégorique, qui est désigné par les mots " puant huillier Caresme », et qui écœure par la « santeur de ses ongnons ». Même lorsque Charnau s'apaise, il ne veut pas que Caresme

\footnotetext{
${ }^{22}$ La Dure Et Cruelle Bataille ..., éd. cit., v. 41-48, p. 5.

${ }^{23}$ Ibid., p. 34. La vision n'est pas sans évoquer le tableau de Brueghel consacré au thème du combat entre Carnaval et Carême. Pour une analyse du tableau, voir Claude GAIGNEBET, « Le combat de carnaval et de Carême de P. Brueghel », Annales ESC., 2 (1972), p. 312-345.

${ }^{24}$ Euvres complètes de Eustache Deschamps, éd. cit., Ballade 350, v. 14-15.
} 
s'approche car il «enput tous le cabas ${ }^{25}$. Ce «cabas» dans lequel on transporte légumes ou poissons constitue d'ailleurs un dernier attribut allégorique de la figure de Caresme, qui tend donc à se fixer, sans se figer, peutêtre en raison de l'élaboration d'un rituel de fête opposant Carnaval et Carême.

Dans ce mouvement de réécriture dynamique du débat se dessine une description plus précise des symptômes de la faim et de l'appétit. Cette dernière notion s'incarne en un personnage, nommé «Appetit-friant»: messager de Charnau, il occupe la fonction symbolique d'intermédiaire entre le corps et la nourriture. L'épithète «friant» attachée à son nom explique sa diligence impétueuse par l'impatience caractéristique du gourmand, et le rattache à la chaleur brûlante et au mouvement frétillant. Le jeu du personnage d'Appétit est donc pétillant, sautillant, comme l'indique l'expression « Tout prompt a ung pas et ung sault $»^{26}$.

Quant aux symptômes du régime maigre, ce sont plutôt ceux de la maladie. La faim est liée aux écoulements d'humeurs, de bile, de «cholere». Caresme fait venir, selon Charnau, « la rongne a la bouche $»^{27}:$ il provoque donc une espèce de gale, mais le terme « rongne » peut également désigner la colère, et les troupes de Caresme, de fait, « enragent de fain ». Les propos conclusifs de Charnau résument en termes médicaux les torts du personnage :

Il use de tresfaulx regime,

Car il n'est ne rongne ne ryme

Ne collericque passion,

Goute, toux, morvë en narine

Dont il ne soit occasion. ${ }^{28}$

\footnotetext{
${ }^{25}$ La Dure Et Cruelle Bataille..., éd. cit., v. 873 p. 53, v. 498 p. 31, v. 11 p. 10, v. 1115 p. 65.

${ }^{26}$ Ibid., v. 698, p. 43. L'éditeur note que cette démarche correspond à la gestuelle traditionnelle du sot.

${ }^{27}$ Ibid., v. 1116, p. 65.

${ }^{28}$ Ibid., v. 1048-1052, p. 62.
} 
La faim est ici associée à tous les actes par lesquels le corps se vide, et on peut également «esternuer de fine fain $»^{29}$. Cet assèchement est directement lié aux maladies rénales, la pierre et la gravelle :

Caresme le trop cruel

Mesmement pour gens gravelleux

Car il fait le Pentagruel

A tous mes notables beuveux. ${ }^{30}$

L'écoulement des humeurs ne s'arrête pas là, et les disciples de Caresme sont également caractérisés par la scatologie ${ }^{31}$, par leur « fain d'aller au retraict $»^{32}$. Directement ou métaphoriquement, la faim expulse du corps l'air, le liquide, le solide: il y a là une image qui fonctionne par inversion, en regard des absorptions répétées de vin auxquelles se livrent les partisans de Charnage.

La faim se manifeste donc non seulement par l'amaigrissement, la concrétion, le rétrécissement du corps, mais également par des formes d'écoulements, par tout ce qui vide et retire de la matière.

Les trois textes réunis ici reposent, malgré leurs différences génériques, sur une même tradition parodique qui se révèle particulièrement créative; sa capacité à susciter un imaginaire renouvelle la représentation et crée de nouvelles associations symboliques. Cette figuration repose sur le moule littéraire du débat et du conflit allégorique, qui introduit sa propre logique : il offre un cadre où l'opposition et le contraste façonnent les deux entités autant que leur développement propre. La faim se dessine progressivement par opposition à l'appétit, et donc comme pulsion du corps vers le vide, comme expulsion des humeurs et dégonflement, alors que le goût de la bonne chère pousse à absorber et à se remplir ; il s'agit là d'une opposition structurante, qui

\footnotetext{
${ }^{29}$ Ibid., v. 1055 , p. 62.

${ }^{30}$ Ibid., v. 93-96, p. 8-9.

${ }^{31}$ Ibid., v. 394-396, p. 25.

${ }^{32}$ Ibid., v. 435, p. 27.
} 
permet de créer une image en utilisant la symétrie et l'inversion, et l'on se gardera de parler de réalisme pour cette représentation profondément liée à une symbolique. 